\title{
Chitin increases the angiogenesis in chorioallantoic membrane model in the presence of testosterone and progesterone
}

\author{
Ezgi İrem Sağlam ${ }^{1}$, İlayda Ceren Kutlu ${ }^{1}$, Orhan Erdem Haberal ${ }^{1}$, Mehmet Yüksekkaya ${ }^{1}$, Özgür Kılıçarslan ${ }^{2}$, \\ Şefik Güran ${ }^{2}$
}

(1) Başkent University, Faculty of Engineering, Biomedical Engineering Program, Ankara, Turkey

(2) University of Health Sciences, Gülhane Faculty of Medicine, Department of Medical Biology, Ankara, Turkey

Date submitted:

Aug 17, 2018

Date accepted:

Sep 28, 2018

Online publication date:

March 15, 2019

\section{Corresponding Author: \\ Prof. Dr. Şefik Güran, \\ Sağlık Bilimleri Üniversitesi, \\ Gülhane Tıp Fakültesi, Tıbbi \\ Biyoloji AD, 0608 Etlik-ANKARA/ \\ TURKEY}

Phone: +903123043551

Keywords: Chitin, testosterone, progesterone, chorioallantoic membrane, angiogenesis, tissue engineering.

\begin{abstract}
Aims:Angiogenesis is a process of generating new blood vessels from preexisting vessels. In adults, it is activated in pathologic conditions. Chitin is an organic molecule which is used in scaffold technology in tissue engineering. Growth hormones such as testosterone and progesterone are used in scaffold structure for induction of angiogenesis. No literature was found about the angiogenic roles of chitin/testosterone/ progesterone. Here, chitin was analyzed in the presence of testosterone/progesterone to find out its' possible role on angiogenesis.

Methods: Chitin was obtained from shrimp shells in our laboratory. The angiogenic effects of chitin/ testosterone/progesterone were analyzed on chick embryo chorioallontoic membrane (CAM) model. Six different study groups were prepared (control group-Group 1, testosterone applied group-Group 2, progesterone applied group-Group 3, chitin/testosterone applied group-Group 4, chitin/progesterone applied group-Group 5, chitin/testosterone/progesterone applied group-Group 6). Hormones were used in different concentrations. The angiogenic role of selected molecules was clarified according to the total differentiation score of angiogenesis (TDSA) results in all groups. In obtaining of TDSA results, Knighton's protocol was applied.

Results: TDSA was $6 \pm 0.1$ in testosterone applied group, $5 \pm 0.2$ in progesterone applied group, $7 \pm 0.1$ in chitin/testosterone applied group, $5 \pm 0.1$ in chitin/progesterone applied group, $7 \pm 0.1$ in chitin/ testosterone/progesterone applied group. In all groups, TDSA results were statistically significant. These results represented the angiogenic role of chitin in the presence of testosterone and progesterone $(p<0.05)$.

Conclusions: Our results support the angiogenic roles of chitin in the presence of testosterone and progesterone. Chitin, testosterone and progesterone can be used in scaffold technology together.
\end{abstract}

\section{Introduction}

Angiogenesis (capillary vascularization) is an interesting process which new blood vessels form from pre-existing vessels. It physiologically occurs only in embryonal period. In adult period, it occurs in pathologic conditions like cancer and wound healing. Because of its positive role in wound healing process, angiogenic factors are used in tissue engineering studies. Different hormones such as testosterone and progesterone are used as angiogenic factors in scaffold technology in artificial skin formation studies $(1,2)$.

Chicken embryo chorioallantoic membrane (CAM) is a complex structure composed of branched blood vessels in chicken eggs. Fertilized eggs CAM part is similar to placenta in mammals. It is a good "in vivo" culture media for the angiogenesis studies $(3,4)$. For this purpose, chicken fertilized embryos have been used in neovascularization studies since the early 1970's (5). Up to now, angiogenic roles of different organic/inorganic molecules had been analyzed in CAM studies. The angiogenic roles of growth factors, hormones, anticancer agents, antibiotics and antibodies were clarified by using CAM in literature (6,
$7,8)$. Today we know that hormones such as testosterone and progesterone stimulate the angiogenesis. Therefore, testosterone and progesterone are used in tissue scaffold structure $(9$, 10).

Chitin is a cationic compound which is found in the shells of sea creatures such as crabs, lobsters and shrimps. It is used in scaffold technology in tissue engineering studies $(11,12)$. Chitosan is a natural fiber which is extracted from chitin. It is made by treating the chitin shells of shrimp and other crustaceans with an alkaline substance, like sodium hydroxide. Chitin and chitosan are used in scaffold technology because of their biodegradable, biocompatible and nontoxic specialties (11). In literature, no manuscript was found about the angiogenic role of chitin in the presence of testosterone and progesterone.

Here, chitin was obtained from shrimp shells in our laboratory. Testosterone and progesterone solutions were prepared in different concentrations. Chitin in the presence of testosterone and progesterone was analyzed by using CAM methodology. Our manuscript is a unique example which explains the angiogenic role of chitin/testosterone/progesterone together in literature. 


\section{Methods}

This study was performed with the ethical decision of University of Health Science, Gülhane Health Science Institute, Animal Experiments Local Ethics Committee (February 27, 2018Etik-2018/06).

Chitin Extraction from Shrimp Shells: Chitin was obtained from shrimp cells in our laboratory by using Kocer's methodology (13). First, shrimp cells were cleaned with distilled water (Figure 1. A). For the deproteinization of shrimp cells, $\mathrm{NaOH}$ solution $(3.5 \% \mathrm{w} / \mathrm{v})$ was used. It was applied on shrimp shells for 2 hours at $65^{\circ} \mathrm{C}(10 \mathrm{ml}$ solution for $1 \mathrm{gr}$ shrimp shell). For the demineralization of shrimp cells, $\mathrm{HCl}$ solution $(1 \mathrm{M})$ was used. It was applied on shrimp shells for 30 minutes at room temperature (15 ml solution for $1 \mathrm{gr}$ shrimp shells). For the neutralization of shells, protein and mineral wastes were removed from the shrimp shells by washing them with distilled water. This neutralization step is important for "in vivo" cell culture studies (14). After neutralization step, chitin molecules were dried in the oven at $60^{\circ} \mathrm{C}$ for one night (Figure 1. B).

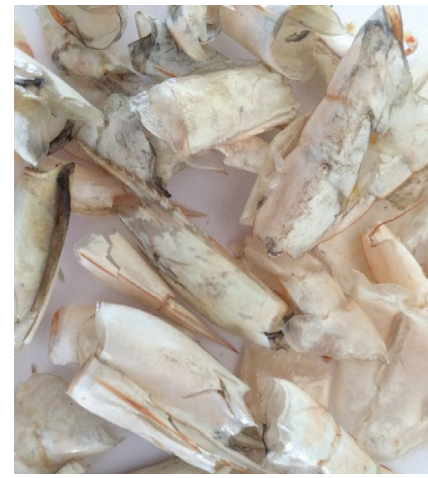

Figure 1A. Shrimp shells which were obtained from shrimps

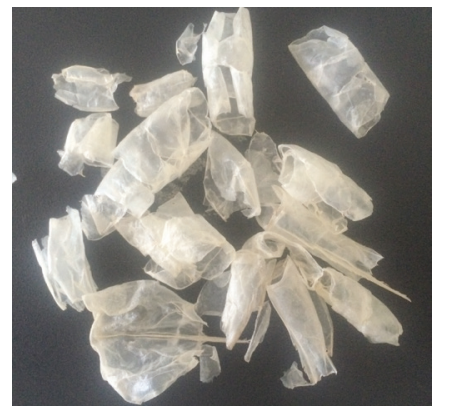

Figure 1B. Chitinswhich were obtained from shrimp cell after deproteinization/demineralization/ neutralization steps.
Preparation of Testosterone and Progesterone Solutions: Testosterone (Sustanon iv. $250 \mathrm{mg} / \mathrm{ml}$ ) was obtained from Schering-Plough Company. Progesterone (Progestan iv. $25 \mathrm{mg} / \mathrm{ml}$ ) was obtained from KocakFarmaCompany. Physiologic serum (SF) was used for dilutions in testosterone and progesterone solutions. Testosterone solutions were prepared in $20 \mathrm{nmol} / \mathrm{L}, 50 \mathrm{nmol} / \mathrm{L}, 100 \mathrm{nmol} / \mathrm{L}$ concentrations (16). Progesterone solution was prepared in $5 \mu \mathrm{mol} / \mathrm{L}, 10 \mu \mathrm{mol} / \mathrm{L}, 20$ $\mu \mathrm{mol} / \mathrm{L}$ concentrations (15).

Preparation of Fertilized Chicken Eggs: Atak-S type fertilized chicken eggs were obtained from the Chicken Production Farm of Başkent University. For our study, one hundred and twenty fertilized chicken eggs were selected carefully. Fertilized chicken eggs were kept one day in a dark room. At the end of 24 hour, they were transferred to aCO2incubator (Sanyo MCO19AIC CO2 170L Incubator). Fertilized chicken eggs were kept hold in an incubator for six days (at $37^{\circ} \mathrm{C}$ heat, $85-90 \%$ humidity). At the end of the 6 th day, the egg shells were cleaned with an antiseptic solution. The shells of eggs were opened carefully with tweezers. As known, vascularization in chick embryo CAM begins at the 5th and the 6th days (17). Therefore, the CAMs of fertilized chicken eggs were visualized and photographed on the 6th day for observing the increase in angiogenesis.

Application of Chitin/testosterone/progesterone on CAM assay: All the fertilized chicken eggs were separated into six different groups for the application of chitin/testosterone/progesterone (Group 1- Control group, Group 2- testosterone applied group, Group 3- progesterone applied group, Group 4- chitin/testosterone applied group, Group 5- chitin/progesterone applied group, Group 6- chitin/testosterone/progesterone applied group). On the 6th day, CAMs were observed and photographed for the neovascularization. Then, SF solution was added as $250 \mu \mathrm{l}$ in control group (Group 1). Chitin was applied in its' natural form (Figure 1. B). Testosterone and progesterone solutions were applied as $250 \mu \mathrm{l}$ for each condition in chitin/hormone applied groups (Groups 2, 3, 4, 5, 6). Then, eggs were covered with parafilm. Parafilm application prevented the eggs from moisture loss and kept the sterilization of the CAMs.At the end, all of the eggs were placed back into the CO2 incubator.

Twenty four hours later (on the 7th day), the parafilms were opened. All the CAMs were analyzed for neovascularization. CAMs were visualized and photographed for observing the changing in angiogenesis.

Methods used in Angiogenesis Measurement:For finding the increase in angiogenesis, Knighton et al.'s scoring methodology was used (17). On the 6th and the 7th days, the increases in vascular structureson CAM were visualized and photographed. Three different observers noted the increases in vascular structure and numbered due to Knighton et al.'s scoring methodology. As known, the observations on the 6th and 7 th days were enough to score the vessels $(17,18,19)$. The response rate in vascular structure was graded as zero $(0)$, one (1), two (2). Zero (0) means no change in vessel formation. One (1) means a minimal increasing in density and length in vessel formation on CAM. Two (2) reflects a maximal increase in density and length in vessel formation on CAM (17). Mean values were obtained from the different observers results. They were reflected the total differentiation score of angiogenesis (TDSA). TDSA results were represented the increase in angiogenesis $(17,18,19)$ (Table 1).

Statistical analyses:TDSAs were obtained by having mean

Table 1. The total differentiation score of angiogenesis (TDSA) results which were seen in control, chitin, testosterone, progesterone applied groups.

\begin{tabular}{|c|c|c|c|c|}
\hline & Study Groups & $\mathbf{n}$ & TDSA & p Values \\
\hline Group 1 & Control & 8 & 0 & \\
\hline Group 2 & Testosterone & 11 & $6 \pm 0.1$ & $p<0.05$ \\
\hline Group 3 & Progesterone & 9 & $5 \pm 0.2$ & $p<0.05$ \\
\hline Group 4 & Chitin + Testosterone & 8 & $7 \pm 0.1$ & $p<0.05$ \\
\hline Group 5 & Chitin + Progesterone & 8 & $5 \pm 0.1$ & $p<0.05$ \\
\hline Group 6 & Chitin + Testosterone +Progesterone & 9 & $7 \pm 0.1$ & $p<0.05$ \\
\hline
\end{tabular}


value results of scores. Student $t$ tests results ( $p$ values) represent two group comparisons of TDSA among control and chitin/ testosterone/progesterone applied groups (Table 1).

\section{Results}

Neutral form of chitin is suitable for "in vivo" studies and/or tissue engineering studies (14). So, neutral form ofchitin was obtained from shrimp shells in appropriate condition and used in our experiments (Figure 1B).

TDSA Results for Testosterone and Progesteroneapplied groups on CAM model: In control group (Group 1), TDSA value was found as zero (0) (Table 1, Figure 2). No obvious increase was observed in CAM assay in 6th and 7 th day results in main and capillary vessel formations (Figure 2, Figure 3A). In testosterone applied group (Group 2), TDSA was found $6 \pm$ 0.1 (Table 1, Figure 2, Figure 3B). TDSA was observed as $5 \pm$ 0.2 in progesterone applied group (Group 3) (Table 1, Figure 2 ). So, testosterone and progesterone increased the main and capillary vesselformations in density and length in eye observations. The increases in TDSA values in Groups 2, 3 emphasize the increase in main and capillary vessel formations as seen in Figure 3B. The TDSA results were statistically significant in groups 2 and 3 ( $p \geq 0.05$ ) (Table 1). Similar TDSA values were observed in the uses of hormones in different concentrations.

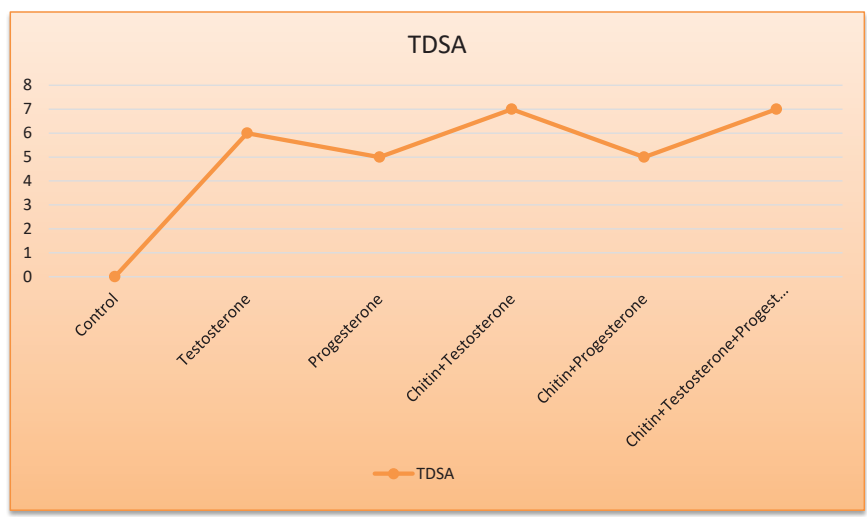

Figure 2. The graphic of total differentiation score of angiogenesis (TDSA) results which were observed in control and chitin/hormone appliedgroups.

Results for Chitin/Testosterone and Chitin/Progesteroneapplied groups on CAM model: In group 4 (chitin/testosterone applied group), TDSA value was found as $7 \pm 0.1$ (Table 1 and Figure 2). This TDSA result represents the angiogenic stimulator role of chitin/testosterone together. Chitin/testosterone increased the main and capillary vessel formations in density and length (Figure $3 C$ ). The TDSA result in group 4 was statistically significant $(p \geq 0.05)$ (Table 1$)$. Similar TDSA values were observed in the uses of testosterone in different concentrations.

In group 5 (chitin/progesterone applied group),TDSA value was found as $5 \pm 0.1$ (Table 1 and Figure 2). High TDSA result represents the angiogenic roles of chitin/progesterone together. Chitin/progesterone also increased the main and capillary vessel formations in density and length on CAM assay. The TDSA resultin group 5 wasalso statistically significant $(p \geq 0.05)$ (Table 1). Similar TDSA values were observed in the uses of testosterone in different concentrations.

Results for Chitin/Testosterone/Progesteroneapplied group on CAM model: In group 6, chitin was used with dif-
Figure 3. CAM assay results which were observed in control and chitin/testosterone/progesteroneappliedgroups.

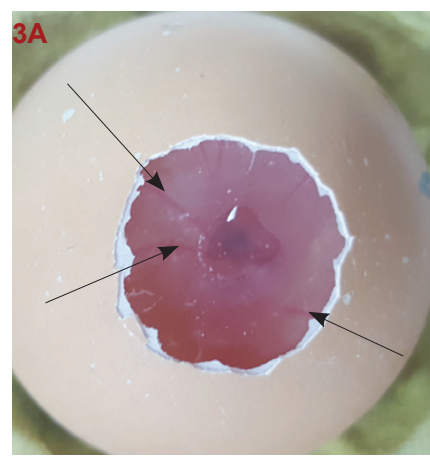

Figure 3A. Control group (Group1) result; arrows indicate minimal increasinginthe main and capillary vessel formations in density and length.

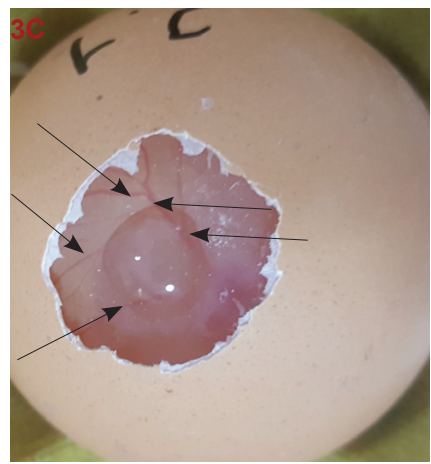

Figure 3C. Chitin/testosterone applied (Group 4) group result; arrows indicate maximal increasing in the main and capillary vessel formations in density and length.

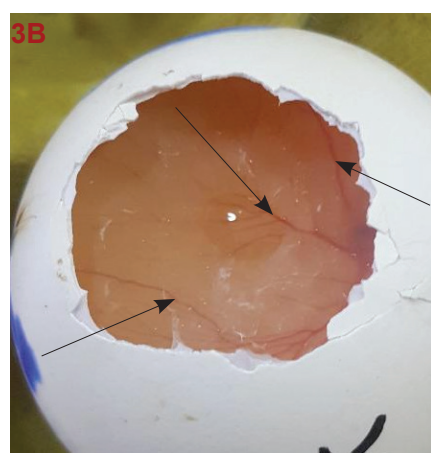

Figure 3B. Testosteroneapplied group (Group 2) result; arrows indicate maximal increasingin the main and capillary vessel formations in density and length.

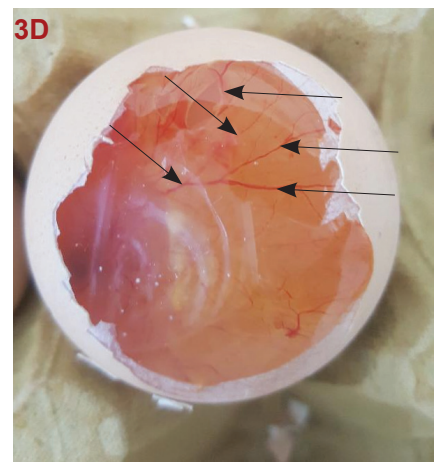

Figure 3D. Chitin/testosterone/progesterone applied(Group 6) group result; arrows indicate maximal increasing in the main and capillary vessel formations in density and length. ferent testosterone/progesterone solutions. TDSA was found as $7 \pm 0.1$ in this group, which represents the stimulator role of chitin/testosterone/progesterone together. Chitin/testosterone/ progesterone treatment increased the main and capillary vessel formations in density and length on CAM assay (Table 1, Figure 2, Figure 3D). The TDSA result obtained in group 6 was statistically significant ( $p \geq 0.05$ ) (Table 1$)$. Similar TDSA values were observed in the uses of testosterone in different concentrations.

We observed that chitin/testosterone/progesterone stimulate angiogenesis on CAM model. In different concentrations of hormones, similar angiogenic effects were found. May be the smallest hormone concentrations used in our experiments (20 nmol/L for testosterone, $5 \mu \mathrm{mol} / \mathrm{L}$ for progesterone) were enough for having angiogenic effect on CAM assay.

\section{Discussion}

Angiogenesis is a process of new vessel formation. Nowadays in medicine it is important to encourage angiogenesis especially for ischemic diseases and wound healing $(20,21)$. Wound healing is a complex and dynamic process of replacing devitalized and missing cellular structures and tissue layers. Angiogenesis plays a crucial role in wound healing by forming new blood vessels from preexisting vessels by invading the wound clot and organizing in to a micro vascular network throughout the granulation tissue. This dynamic process is highly regulated by signals from both serum and the surrounding extracellular 
matrix environment. Vascular endothelial growth factor, angiopoietin, fibroblast growth factor and transforming growth factor beta are amongst the potentangiogenic cytokines in wound angiogenesis (22). Therefore, potent angiogenic factors generally use in tissue regeneration studies in tissue engineering and regenerative medicine (especially in manufacturing of artificial skin) (23). Today, growth factors are used in scaffold structure for stimulating the angiogenesis in wound healing studies. In our experiment, testosterone and progesterone were analyzed for angiogenesis with chitin which one generally uses in scaffolds structure in tissue engineering (24).

CAM model of chick embryos is a useful in-vivo working model for angiogenesis because of its' high sensitivity (25). In literature, we know that the hormones and growth factors stimulate angiogenesis in certain tissues $(26,27)$. Despite of these findings, no study has been observed in literature about the angiogenic role of chitin/testosterone/progesterone together. In our manuscript, the possible positive angiogenic effects of testosterone and progesterone were analyzed on CAM in study groups 2 and 3 (only testosterone or only progesterone). We observed the stimulatory effects of testosterone/progesterone hormones which support literature findings (Table 1, Figure 2, Figure 3B) $(26,27)$.

Chitin is abundant in invertebrates and fungi as an important structural molecule. It is a primary component of cell walls in fungi, the exoskeletons of arthropods, such as crabs, lobsters and shrimps and insects $(27,28)$. Chitin has proved useful for several medicinal, industrial and biotechnological purposes. In biotechnology, it generally uses in scaffold structure (29). In our experiments, we obtained chitin from shrimp shells in our laboratory (Figure 1A). At the end, we neutralized them with distilled water. In this way they were formed as useful for "in vivo" studies (Figure 1B).

The angiogenic role of chitin in the presenceof testosterone/ progesterone was analyzed in study groups 4 and 5(Chitin and testosterone or progesterone). Excessive vessel formations were observed on CAM in this panel (Table 1, Figure $3 \mathrm{C}$ ). In group 6 (Chitin and testosterone and progesterone)angiogenesis was increased by chitin in the presenceof testosterone/ progesterone (Table 1, Figure 3D). Our manuscript is the first example in literature which represents angiogenic role of chitin/ testosterone/progesterone together.

In our study, testosterone and progesterone hormones were used in different concentrations. Similar results were obtained in highest and lowest hormone concentrations. No extra angiogenic role was observed in high hormone dosages in hormone and chitin/hormone groups (Groups 2, 3, 4, 5, 6). We suppose that the lowest hormone dosages used in our experiments were enough in angiogenic role on CAM.

Chick embryo CAM model results support angiogenic roles of chitin, testosterone and progesterone. Chitin can be used easily in scaffold technology with testosterone and progesterone.

\section{Acknowledgements}

Study was designed by EIS, ICK, OEH, MY and ŞG. Data was collected and analyzed by EIS, ICK, ÖK ŞG. Manuscript was written by EIS, ICK, OEH, MY and ŞG. There was no financial assistance with the project.

\section{Conflict of Interest}

No conflict of interest exists related to our submission.

\section{References}

1. Carmeliet $P$, Jain RK. Molecular mechanisms and clinical applications of angiogenesis. Nature. 2011; 473(4):298307.

2. Ud-Din S, Sebastian A, Giddings P, et al. Angiogenesis is induced, and wound size is reduced by electrical stimulation in an acute wound healing model in human skin. PLoS One. 2015;10(4):e0124502.

3. Birbrair A, Zhang T, Wang ZM, Messi ML, Mintz A, Delbono $O$. Pericytes at the intersection between tissue regeneration and pathology. Clinical Science 2015; 128 (2): 81-93.

4. Fáncsi T, Fehér G. Ultrastructural studies of chicken embryo chorioallantoic membrane during incubation. Anat Histol Embryol. 1979; 8(2): 151-159.

5. Auerbach R, Kubai L, Knighton D, Folkman J. A simple procedure for the long-term cultivation of chicken embryos. Dev Biol. 1974; 41(2): 391-394.

6. Guran S, Çoban ZD, Fermanli O, Aydıngöz E, Ipek E. Folic acid and zinc inhibits angiogenesis in chicken CAM model via angiogenic factor genes. Gulhane Med. Journal (in press).

7. Tufan AC, Satiroglu-Tufan NL. The chick embryo chorioallantoic membrane as a model system for the study of tumor angiogenesis, invasion and development of anti-angiogenic agents. Curr Cancer Drug Targets 2005; 5(4):249-266.

8. Nowak-Sliwinska P, Segura T, Iruela-Arispe ML. The chicken chorioallantoic membrane model in biology, medicine and bioengineering. Angiogenesis 2014; 17 (4):779-804.

9. Chen G, Ushida T, Tateishi T. Scaffold Design for Tissue Engineering. Macromol. Biosci. 2002; 2(2): 67-77.

10. Brochhausen C, Lehmann M, Halstenberg S, Meurer A, Klaus G, Kirkpatrick CJ.J. Signalling molecules and growth factors for tissue engineering of cartilage-what can we learn from the growth plate? Tissue Eng Regen Med. 2009;3(6):416-429.

11. Oryan A, Sahvieh S. Effectiveness of chitosan scaffold in skin, bone and cartilage healing. Int J Biol Macromol. 2017;104(Pt A):1003-1011.

12. Ji WC, Zhang XW, Qiu YS. Selected suitable seed cell, scaffold and growth factor could maximize the repair effect using tissue engineering method in spinal cord injury. World J Exp Med. 2016;6(3):58-62.

13. Kocer I. [The isolation and characterization of Chitosan in different ways]. Hacettepe University Science Institute, Master's thesis,Ankara, 2015.

14. No HK, Meyers SP, Lee KS. Isolation and characterization of chitin from crawfish shell waste, Journal of Agricultural and Food Chemistry 1989; 37 (4): 575-579.

15. Waheed S, Dorjbal B, Hamilton CA, Maxwell GL, Rodriguez GC, Syed V. Progesterone and calcitriol reduce invasive potential of endometrial cancer cells by targeting ARF6, NEDD9 and MT1-MMP. Oncotarget. 2017; 8(69): 113583-113597. 
16. Holmes S, Singh M, Su C, Cunningham RL. Effects of oxidative stress and testosterone on pro-inflammatory signaling in a female rat dopaminergic neuronal cell line. Endocrinology 2016; 157(7): 2824-2835.

17. Knighton D, Ausprunk D, Tapper D, et al: Avascular and vascular phases of tumour growth in the chick embryo. Br J Cancer 1977;35(3);347-356.

18. Özgürtaş T. An in vivo model for angiogenesis: chick chorioallantoic membrane. Gulhane Medical Journal 2009; 51(1): 67-69.

19. Gülsen RM, Uzunay SN, Fermanlı O, et al. Anti-angiogenic role of Ankaferd on chick chorioallontoic membrane model. Gulhane Med J. 2015; 57(3): 274-279.

20. Stewart DJ, Hilton JD, Arnold JM, et al. Angiogenic gene therapy in patients with non revascularizable is chemic heart disease: a phase 2 randomized, controlledtrial of AdVEGF(121) (AdVEGF121) versus maximum Medical treatment. Gene Ther. 2006;13(21):1503-1511.

21. Yuan R, Xin Q, Shi W, et al. Vascular Endothelial Growth Factor Gene Transfer Therapy for Coronary Artery Disease: A Systematic Review and Meta-Analysis. Cardiovasc Ther. 2018 Jul 23:e12461.

22. Honnegowda TM, Kumar P, Udupa EG, Kumar S, Kumar $U$, Rao P. Role of angiogenesis and angiogenic factors in acute and chronic wound healing. Plast Aesthet Res 2015; 2(4): 243-249.

23. Silva SS, Caridade SG, Mano JF, Reis RL. Effect of crosslinking in chitosan/aloevera-basedmembranesforbiomedicalapplications.CarbohydrPolym. 2013; 98(1): 581-588.

24. Santos TC, Höring B, Reise $K$, et al. In vivo performance of chitosan/soy-based membranes as wound-dressing devices for acute skin wounds. Tissue Eng Part A. 2013;19(7-8):860-869.

25. Ribatti $D$. The chickembryo chorioallantoic membrane in the study of tumor angiogenesis. Rom J Morphol Embryol. 2008; 49(2): 131-135.

26. Chodari L, Mohammadi M, Ghorbanzadeh V, Dariushnejad H, Mohaddes G. Testosterone and Voluntary Exercise Promote Angiogenesis in Hearts of Rats with Diabetes by Enhancing Expression of VEGF-A and SDF-1a. Can J Diabetes 2016;40(5):436-441.

27. Yu P, Li S, Zhang Z, Wen X, et al. Progesterone-mediated angiogenic activity of endothelial progenitor cell and angiogenesis in traumatic brain injury rats were antagonized by progesterone receptor antagonist. Cell Prolif. 2017; 50(5): 321-329.

28. Tang WJ, Fernandez JG, Sohn JJ, Amemiya CT. Chitin is endogenously produced in vertebrates. Curr Biol. 2015; 25(4): 897-900.

29. Ifuku S. Chitin and Chitosan Nanofibers: Preparation and Chemical Modifications. Molecules 2015; 19(11): 18367-18380. 